\title{
Transplantation of cryopreserved muscle cells in dilated cardiomyopathy: Effects on left ventricular geometry and function
}

\author{
Nobuhisa Ohno, MD \\ Paul W. M. Fedak, MD \\ Richard D. Weisel, MD \\ Donald A. G. Mickle, MD \\ Takeshiro Fujii, MD \\ Ren-Ke Li, MD, PhD
}

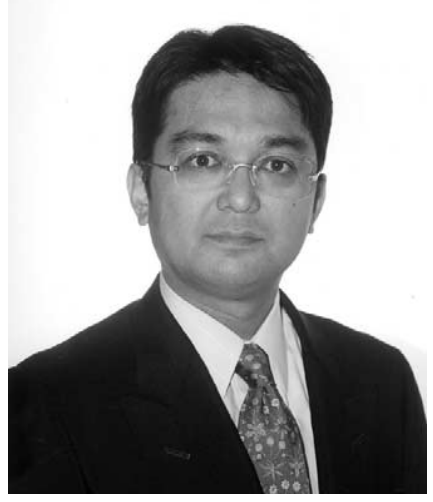

Dr Ohno

\begin{abstract}
From the Division of Cardiac Surgery, Department of Surgery, The Toronto General Hospital, University of Toronto, Toronto, Ontario, Canada.

Ren-Ke $\mathrm{Li}$ is a Career Investigator of the Heart and Stroke Foundation of Canada. Paul W. M. Fedak is a Research Fellow of the Canadian Institute for Health Research and the Heart and Stroke Foundation of Canada. This research was supported by $\mathrm{Dr}$ Li's research grant funded by the Heart and Stroke Foundation of Ontario (NA\#4603 and NA\#4829) and the Canadian Institute of Health Research (MOP14795).

Received for publication Sept 10, 2002; revisions requested Oct 25, 2002; revisions received June 4, 2003; accepted for publication June 18, 2003.

Address for reprints: Ren-Ke Li, MD, PhD, The Toronto General Hospital, NUI-115, 200 Elizabeth St, Toronto, Ontario, Canada, M5G 2C4 (E-mail: Renke.Li@ uhn.on.ca).

J Thorac Cardiovasc Surg 2003;126: 1537-48

Copyright (C) 2003 by The American Association for Thoracic Surgery

$0022-5223 / 2003 \$ 30.00+0$

doi:10.1016/S0022-5223(03)01021-3
\end{abstract}

Objective: Cell transplantation to prevent congestive heart failure in patients with inherited dilated cardiomyopathy might require the use of noncardiac donor cells unaffected by the genetic defect and cryopreservation to permit cell storage until the time of transplantation. However, the effects of cryopreservation on peripheral muscle cells harvested from a cardiomyopathic recipient and their subsequent ability to restore cardiac structure and function after transplantation are unknown.

Methods: Skeletal myoblasts and vascular smooth muscle cells from cardiomyopathic hamsters ( $\delta$-sarcoglycan-deficient BIO 53.58 hamster) and age-matched normal donor hamsters were isolated, expanded in culture, and cryopreserved. After reanimation in culture, cell morphology and growth rate were assessed and compared with values seen in noncryopreserved cells. A total of $4 \times 10^{6}$ previously cryopreserved skeletal myoblasts $(\mathrm{n}=10)$ and vascular smooth muscle cells $(\mathrm{n}=$ 10) harvested from cardiomyopathic donors were then transplanted into the left ventricles of 17-week-old BIO 53.58 hamsters. Hearts injected with culture medium alone $(\mathrm{n}=11)$ served as controls. Heart function was assessed 5 weeks after transplantation on a Langendorff apparatus, and left ventricular geometry was quantified by means of computerized planimetry. Staining with 5-bromo-2'-deoxyuridine identified the injected cells.

Results: Vascular smooth muscle cells from cardiomyopathic donors had an abnormal morphology and diminished growth rates in culture compared with vascular smooth muscle cells from normal donors. These markers of injury were exacerbated by cryopreservation. In contrast, vascular smooth muscle cells from normal donors and skeletal myoblasts from either cardiomyopathic or normal donors appeared normal in culture and were unaffected by cryopreservation. Both cryopreserved vascular smooth muscle cells and skeletal myoblasts from cardiomyopathic donors formed a viable muscle-resembling tissue that prevented wall thinning, limited left ventricular dilatation, and preserved global systolic function in hamsters with a genetic dilated cardiomyopathy. However, attenuation of cardiac remodeling and preservation of global heart function was greater after skeletal myoblast transplantation compared with vascular smooth muscle cell transplantation in parallel to the in vitro morphologic and growth characteristics of these cells.

Conclusions: Cryostorage of healthy donor cells does not prevent the benefits of cell transplantation on limiting remodeling and preserving cardiac function in the failing heart. The health of donor cells in vitro predicts their subsequent benefits on cardiac structure and function after transplantation. Cryopreservation of donor cells might facilitate a clinically applicable and effective approach for ventricular restoration with cell-transplantation therapy for patients with inherited dilated cardiomyopathy. 


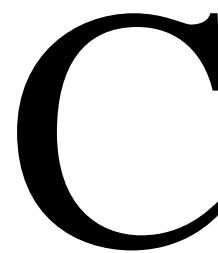

ell transplantation has been demonstrated to prevent cardiac dilatation and dysfunction after myocardial injury, such as that caused by coronary ischemia, infarction, or local cryoinjury. ${ }^{1-6}$ In addition, we and others found that cell transplantation preserved ventricular function in animals with a progressive dilated cardiomyopathy. ${ }^{7-9}$ However, the clinical applicability of these encouraging early results was limited by the cell types used and the subsequent timing of transplantation. First, given the global nature of the disease, cardiac muscle cells might be abnormal in patients with a dilated cardiomyopathy. Extracardiac muscle cells, such as peripheral skeletal muscle cells (SKMCs) or vascular smooth muscle cells (VSMCs), might eliminate the need for transplantation of diseased cardiac cells. Second, in our previous studies the duration from cell harvest to cell transplantation was not flexible and was determined by the growth rate of the cells in culture. Cryopreservation might provide a safe method for donor cell storage, allowing for targeted cell therapy, but no study to date has examined the effects of cryopreservation on the ability of donor cells to restore cardiac structure and function. In addition, it is not known whether the growth characteristics of cells in culture are predictive of their subsequent benefits after transplantation. The ability to identify cells in culture that are capable of providing benefits after cell transplantation, particularly after a period of prolonged storage, would facilitate and optimize clinical cell-transplantation therapies.

The objectives of this study were 2-fold. First, we aimed to establish a proof of concept that peripheral muscle cells can be safely cryopreserved, reanimated in culture, and then transplanted into cardiomyopathic hearts, resulting in improved left ventricular (LV) structure and function. Second, the in vitro characteristics of the cells were assessed to evaluate tolerance to cryostorage and also to determine the feasibility of using in vitro characteristics to predict subsequent in vivo effects after cryostorage and transplantation.

\section{Material and Methods \\ Experimental Design}

The design of this study is described in Figure 1. First, the differential effects of cryostorage on SKMC and VSMC survival, growth, and morphology in culture were assessed from cardiomyopathic hamsters with an underlying genetic defect ( $\delta$-sarcoglycandeficient BIO 53.58 hamsters) versus similar cells from normal hamster donors. Second, the cryopreserved SKMCs and VSMCs from cardiomyopathic donors were transplanted into 17 -week-old cardiomyopathic recipient hamsters to evaluate the ability of myopathic cells to improve LV structure and function after a period of cryostorage and to simulate a clinical scenario. The in vitro characteristics of the cells were then compared with their subsequent ability to restore ventricular function to determine the feasibility of using in vitro characteristics to predict subsequent in vivo effects after cryostorage and transplantation.

\section{Experimental Animals}

All procedures and protocols were approved by the Animal Care Committee of the University Health Network (Toronto, Ontario, Canada). Male BIO 53.58 hamsters (BIO Breeders) and normal Mongolian hamsters were used in the study. Donor muscle cells were freshly isolated from 4-week old BIO 53.58 hamsters. Seventeen-week-old BIO 53.58 hamsters were used as recipients. All experiments were performed in accordance with the "Guide to the Care and Use of Experimental Animals" of the Canadian Council on Animal Care" and the "Guide of the Care and Use of Laboratory Animals" (National Institutes of Health publication no. 85-23, revised 1985).

The animals were divided into 4 groups according to the specific procedure performed: SKMC transplantation (SKMC group, $\mathrm{n}=10$ ), VSMC transplantation (VSMC group, $\mathrm{n}=10$ ), culture medium injected without donor cells (control group, $\mathrm{n}=$ 11 ), and age-matched normal hamsters without any surgical intervention (normal group, $\mathrm{n}=7$ ).

\section{Isolation, Culture, Cryopreservation, and Identification of Donor Cells}

Cell isolation and culture. Biopsy specimens of the skeletal muscle and aorta were collected from donor hamsters (4-week-old BIO 53.58 hamsters). Immediately after being killed, the bilateral thigh muscles and the whole aorta were excised and processed for cell culture. One hamster provided approximately 4 transplantation donor cells. The thigh muscle was minced and incubated in $20 \mathrm{~mL}$ of phosphate-buffered saline (PBS; $\mathrm{NaCl}, 136.9 \mathrm{mmol} / \mathrm{L} ; \mathrm{KCl}, 2.7$ $\mathrm{mmol} / \mathrm{L} ; \mathrm{Ma}_{2} \mathrm{HPO}_{4}, 8.1 \mathrm{mmol} / \mathrm{L} ; \mathrm{KH}_{2} \mathrm{PO}_{4}, 1.5 \mathrm{mmol} / \mathrm{L} ; \mathrm{pH} 7.3$ ) containing $0.2 \%$ Type I collagenase (Sigma) and $0.02 \%$ glucose at $37^{\circ} \mathrm{C}$ for 15 minutes. After centrifugation at $600 \mathrm{~g}$ for 5 minutes at room temperature, the cell pellet was isolated, and the supernatant was discarded. The tissue pellet was then digested with $40 \mathrm{~mL}$ of PBS containing $0.15 \%$ pronase (Sigma) for 10 minutes at $37^{\circ} \mathrm{C}$. Cell culture medium (M-199, Gibco) containing $10 \%$ fetal bovine serum (FBS; Gibco), $100 \mathrm{U} / \mathrm{mL}$ penicillin, and $100 \mathrm{mg} / \mathrm{mL}$ streptomycin was added to the cell containing the supernatant to neutralize enzymatic activity. After centrifugation, cells were preplated on a noncoated culture dish for 30 minutes to try to purify the cell pellet as much as possible. Then the supernatant, which contained a higher cell population of satellite cells than before preplating, was transferred into $60-\mathrm{mm}$ laminin-coated culture dishes (Becton Dickinson). The SKMCs were subcultured once during the period of culturing (5-6 days) before cryopreservation. Satellite cells had to be subcultured in approximately $60 \%$ confluence to prevent them from forming myotubes in culture. As a result, the culture period was shorter in SKMCs than VSMCs.

VSMCs were isolated as previously described. ${ }^{7}$ In brief, 0.5 $\mathrm{mL}$ of PBS containing $0.2 \%$ Type II collagenase (Worthington), $0.4 \%$ trypsin (DIFCO), and $0.02 \%$ glucose was injected into the aortic lumen and incubated for 20 minutes at $37^{\circ} \mathrm{C}$. The lumen was flushed with PBS to remove endothelial cells. The aorta was minced and incubated in $12.5 \mathrm{~mL}$ of the same enzyme for 15 minutes at $37^{\circ} \mathrm{C}$. The cells in the supernatant were transferred into Iscove's modified Dulbecco's cell culture medium (IMDM; Gibco) containing 10\% FBS, $0.1 \mathrm{mmol} / \mathrm{L} \beta$-mercaptoethanol, 100 $\mathrm{U} / \mathrm{mL}$ penicillin, and $100 \mathrm{mg} / \mathrm{mL}$ streptomycin. After centrifugation, the cell pellet was resuspended in the culture medium and 


Part A
$\begin{aligned} & \text { No } \\ & \text { Noll }\end{aligned}$
Cryostorage

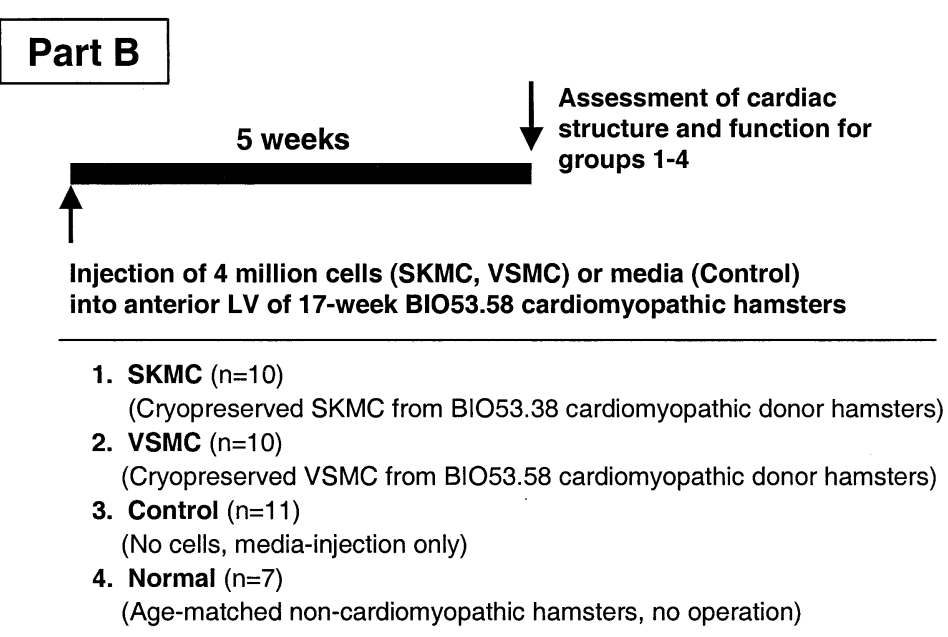

Figure 1. Experimental design. A, differential in vitro effects of cryopreservation on muscle cell growth and morphology from myopathic versus normal donor hamsters. Open bars indicate cells from normal hamsters, and shaded bars indicate cells from cardiomyopathic BI053.58 hamsters. B, In vivo effects of muscle cell transplantation from cardiomyopathic donors for use in dilated cardiomyopathy after prior cryopreservation.

plated into a 10-mm culture dish. The VSMCs were subcultured once during the period of culturing (14 days) before cryopreservation. Before each subculture and transplantation, VSMCs reached approximately $80 \%$ confluence. The VSMCs expanded less rapidly in culture compared with the SKMCs and were maintained longer in culture to achieve equal cell numbers in both groups.

Cryopreservation. When the cells in passage 2 reached certain confluence (60\% in SKMCs and $80 \%$ in VSMCs), the cells were cryopreserved, as previously reported. ${ }^{10}$ Briefly, the cultured cells were washed with PBS and then detached from the culture dish and separated from each other by means of incubation with $0.05 \%$ trypsin in PBS for 2 minutes. After the addition of culture medium (M-199 for SKMCs and IMDM for VSMCs) containing 10\% FBS, the cell suspension was centrifuged. The cell pellet was then resuspended in the cryopreservation solution (10\% dimethylsulf- oxide, 20\% FBS, and $70 \%$ culture medium) and stored in cryovials. The cryovials were then frozen and stored in liquid nitrogen for a 3-week period. Before transplantation, cryopreserved cells were thawed quickly in a water bath at $37^{\circ} \mathrm{C}$ and plated on a laminin-coated dish with M-199 containing 10\% FBS for SKMCs $\left(5 \times 10^{3}\right.$ cells $\left./ \mathrm{cm}^{2}\right)$ or on an uncoated dish with IMDM containing $10 \%$ FBS for VSMCs $\left(2.6 \times 10^{3}\right.$ cells $\left./ \mathrm{cm}^{2}\right)$. The cells were cultured until the time of transplantation ( 3 days for SKMCs and 7 days for VSMCs).

Cell identification. Approximately $2 \%$ of the cultured SKMCs were kept in the cell culture incubator after thawing to identify myotube formation. The cultured SKMCs and VSMCs were identified immunohistochemically by using monoclonal anti-MyoD1 antibody (Dako) and anti- $\alpha$-smooth muscle actin antibody (Sigma), respectively, as previously described. ${ }^{3}$ 


\begin{abstract}
Assessment of Cell Growth
Cell growth rates were compared among cryopreserved and noncryopreserved cells from cardiomyopathic hamsters (BIO) and normal hamsters to determine the effect of cell type on growth. This comparison was done for both VSMCs and SKMCs (BIO VSMCs, $\mathrm{n}=4$; normal VSMCs, $\mathrm{n}=4$; BIO SKMCs, $\mathrm{n}=4$; normal SKMCs, $n=4)$. Because passages before and after cryopreservation correspond to passage 2 and passage 3 , respectively, growth rates before and after cryopreservation were compared with those in passage 2 and passage 3 of noncryopreserved cells. Cell growth rate was calculated at passage 2 (before cryopreservation) and 3 (after cryopreservation), and it was expressed as doubling time.
\end{abstract}

\section{Identification of the Transplanted Donor Cells In Vivo} The cultured SKMCs $(n=2)$ and VSMCs $(n=2)$ were labeled with the thymidine analog 5-bromo-2'-deoxyuridine (BrdU) before transplantation to identify the transplanted cells in the recipient myocardium. When the cells reached $50 \%$ confluence, $25 \mathrm{~mL}$ of $0.4 \% \mathrm{BrdU}$ solution was added to culture dishes and incubated for 48 hours before transplantation. After cell counting, the proportion of BrdU-incorporated cells was adjusted to $15 \%$ for each cell injection.

\section{Cell Preparation and Transplantation}

Cell preparation. The cultured cells were washed 3 times with PBS to remove dead cells and debris. The cells were then detached from the cell culture dish and separated by adding $0.05 \%$ trypsin in PBS to the culture dish for 2 minutes. After the addition of 10 $\mathrm{mL}$ of culture medium, the cell suspension was centrifuged at $580 \mathrm{~g}$ for 5 minutes. The cell pellet was then resuspended in culture medium to reach a concentration of $100 \times 10^{6}$ cells $/ \mathrm{mL}$. A volume of $0.04 \mathrm{~mL}$ of cell suspension or culture medium alone was then transplanted into each heart.

Cell transplantation. The recipient hamsters were anesthetized with ketamine $(20 \mathrm{mg} / \mathrm{kg}$ body weight intramuscularly), followed by an intraperitoneal injection of pentobarbital $(30 \mathrm{mg} / \mathrm{kg}$ body weight). The anesthetized hamsters were intubated, and positive pressure ventilation was maintained with a Harvard ventilator (Model 683, South Natick). The respiratory rate was set at 60 cycles/min, with a tidal volume of $1.5 \mathrm{~mL}$ of oxygen-supplemented room air. The heart was exposed through a $3-\mathrm{cm}$ left lateral thoracotomy. By using a tuberculin syringe, a single injection on the surface of the anterior LV free wall delivered $4 \times 10^{6}$ cells in a $0.04-\mathrm{mL}$ total volume. The needle was inserted into the apex and tracked up toward the base of the heart. The cells were slowly released while the needle tip was withdrawn, leaving cells throughout the anterolateral left ventricle from base to apex between the left anterior coronary artery and the great cardiac vein on the posterior wall. The transplant area covered approximately $40 \%$ of the surface of the anterolateral left ventricle in all animals injected. Control animals were injected with culture medium by using the same technique as used for the cell-transplanted animals. The muscle layer and skin incision were closed with 5-0 vicryl sutures. The hamsters recovered from the operation in a warm environment and were electrocardiographically monitored for 4 hours. Chloramphenicol (Chloromycetin; Parke-Davis, $0.1 \mathrm{mg} / \mathrm{kg}$ administered intramuscularly) and buprenorphine $(0.01-0.05 \mathrm{mg} / \mathrm{kg}$ adminis- tered subcutaneously) were given for 2 days after the operation. Cyclosporine, at a dose of $5 \mathrm{mg} / \mathrm{kg}$ body weight per day, was administered subcutaneously to all groups each postoperative day. The hamsters were housed in cages with filter tops.

\section{Determination of LV Function}

Five weeks after cell transplantation, heart function was evaluated in all groups by using a Langendorff preparation. The hamsters were anesthetized, and heparin sodium (100 units) was administered intravenously. The heart was quickly isolated and perfused in a Langendorff apparatus with filtered Krebs-Henseleit buffer $\left(\mathrm{NaCl}, 118 \mathrm{mmol} / \mathrm{L} ; \mathrm{KCl}, 4.7 \mathrm{mmol} / \mathrm{L} ; \mathrm{KH}_{2} \mathrm{PO}_{4}, 1.2 \mathrm{mmol} / \mathrm{L}\right.$; $\mathrm{CaCl}_{2}, 2.5 \mathrm{mmol} / \mathrm{L} ; \mathrm{MgSO}_{4}, 1.2 \mathrm{mmol} / \mathrm{L} ; \mathrm{NaHCO}_{3}, 25 \mathrm{mmol} / \mathrm{L}$; and glucose, $11 \mathrm{mmol} / \mathrm{L} ; \mathrm{pH}$ 7.4) equilibrated with $5 \%$ carbon dioxide and $95 \%$ oxygen. A latex balloon was passed into the left ventricle through the mitral valve and connected to a pressure transducer (model p10EZ, Viggo-Spectramed) and differentiator amplifier (model 11-G4113-01, Gould Instrument System Inc). After 30 minutes of stabilization, the balloon size was increased by 0.005 - $\mathrm{mL}$ increments from $0.005 \mathrm{~mL}$ to $0.065 \mathrm{~mL}$ by means of the stepwise addition of saline. Coronary flow was measured in triplicate by means of timed collection in the empty beating state. After measuring coronary flow for 1 minute, heart rate and systolic and diastolic LV pressures were recorded at each balloon volume. Developed pressure was calculated as the difference between the systolic and diastolic pressure. After completion of the measurements, the hearts were arrested with coronary perfusion of $5 \mathrm{~mL}$ of $20 \% \mathrm{KCl}$ solution. The passive diastolic pressure in the arrested heart was recorded at each balloon volume in $0.005-\mathrm{mL}$ increments from $0.005 \mathrm{~mL}$ to $0.09 \mathrm{~mL}$ by means of the stepwise addition of saline. After evaluation of cardiac function, hearts were weighed and fixed with $10 \%$ formaldehyde for histology.

\section{Geometric Mapping of the Left Ventricle}

The LV volume was measured, as previously described. ${ }^{7,8,11,12}$ Briefly, the hearts were fixed in LV distension $(30 \mathrm{~mm} \mathrm{Hg})$ with $10 \%$ phosphate-buffered formalin for 48 hours and sectioned in the short axis (1-mm thickness) to yield 6 segments. The segments were photographed and quantified by using computerized planimetry (Jandal Scientific Sigma-Scan). Both the apical and the basal sides of each segment were evaluated. The luminal area of the left ventricle for each segment was determined by calculating the area as outlined by the endocardial circumference. The mean luminal area was determined by calculating the mean luminal area for both the apical and basal sides of each segment. The total LV volume was calculated as the sum of the luminal area for each segment multiplied by the uniform thickness of $1 \mathrm{~mm}$. The average of the LV anteroposterior diameter and the septolateral diameter at the midpapillary section was calculated. The average of 4 opposite LV walls (anterior, posterior, septal, and lateral) was also calculated. The ratio of LV wall thickness to LV diameter was calculated.

\section{Histology}

After planimetry, heart segments were embedded in paraffin, sectioned at a $10-\mu \mathrm{m}$ thickness, and mounted on glass slides. Hematoxylin and eosin and elastica Masson trichrome staining were performed, as described by the manufacturer's specifications 
(Sigma). Immunohistochemical staining ${ }^{3}$ was performed for BrdU, muscle-specific actin (Dako), skeletal fast myosin heavy chain (Sigma), cardiac-specific $\beta$-myosin heavy chain (Chemicon), and $\alpha$-smooth muscle actin (Sigma).

\section{Statistical Analysis}

Results are presented as means \pm SD. SAS system software was used for all analyses (SAS Institute). For the cell-growth study and geometric analysis, comparisons of continuous variables among more than 2 groups were performed by using 1-way factorial analysis of variance. If the F ratio was significant, the Scheffe test was used to specify differences between groups. Function data were evaluated for the 4 groups by means of analysis of covariance by using intraballoon volume as covariance and peak systolic pressure, developed pressure, and end-diastolic pressure as dependent variables. Main effects were group, balloon volume, and interaction of group by balloon volume. If a significant difference was identified, multiple pairwise comparisons were performed to specify between-group differences.

\section{Results}

\section{In Vitro Cell Growth}

The rate of cell growth in vitro was evaluated by means of serial cell counting and calculating the doubling time (Table 1). In normal hamsters smooth muscle cells grew faster than SKMCs ( $P=.008$ at passage 2$)$. However, in cardiomyopathic hamsters the capacity for smooth muscle cells to proliferate was altered. In cardiomyopathic hamsters VSMCs grew more slowly than SKMCs $(P=.003$ at passage 2 and $P=.02$ at P3). We next evaluated the effects of prior cryopreservation and cell storage on the subsequent growth rate of the cells after thawing and reanimation in culture. Cryopreservation had a profound effect on limiting the growth rate of the VSMCs isolated from cardiomyopathic donors. Interestingly, VSMCs isolated from normal hamsters without the genetic cardiomyopathy were not affected by prior cryopreservation. SKMCs taken from either normal or cardiomyopathic donor hamsters were not significantly influenced by cryopreservation.

\section{In Vitro Cell Morphology and Identification}

To further assess the suitability of these muscle cells for transplantation, we next examined their morphologic characteristics and the expression of cell-specific markers by means of immunohistochemistry. Before cryopreservation, the cardiomyopathic VSMCs were larger than the SKMCs (Figure 2). In addition, the morphology of the VSMCs from cardiomyopathic donors contrasted with the smaller spindle-shaped appearance of the smooth muscle cells from normal donors, indicating that the health of these cells was compromised even before cryopreservation. Also in keeping with the cell proliferation data, no morphologic differences were noted between the SKMCs from normal and
TABLE 1. Doubling time of donor cells

\begin{tabular}{llc}
\hline A. Doubling time (hours) & & \\
\hline & \multicolumn{1}{c}{ P2 } & P3 \\
\hline SKMC normal & $56 \pm 12$ & $50 \pm 4$ \\
SKMC BIO & $35 \pm 17$ & $50 \pm 1$ \\
VSMC normal & $21 \pm 6$ & $36 \pm 13$ \\
VSMC BIO & $60 \pm 12^{*}$ & $159 \pm 50 \dagger$
\end{tabular}

B. Effect of prior cryopreservation at passage 2 on doubling time (hours) after thawing at passage 3

\begin{tabular}{lcc}
\hline & Noncryopreserved & Cryopreserved \\
\hline SKMC normal & $50 \pm 4$ & $76 \pm 5$ \\
SKMC BIO & $50 \pm 1$ & $60 \pm 17$ \\
VSMC normal & $36 \pm 3$ & $40 \pm 20$ \\
VSMC BIO & $159 \pm 50$ & $271 \pm 59 \ddagger$
\end{tabular}

$P$, Passage.

${ }^{*} P<.001$ versus normal VSMC at $P 2$.

$\dagger P=.01$ versus normal VSMC at $\mathrm{P} 3$.

$\ddagger P<.001$ versus noncryopreserved BIO VSMC at P3.

cardiomyopathic donors, suggesting that these cells were healthy in culture before cryopreservation. After cryopreservation and before transplantation, the purity of the cells was assessed by means of immunohistochemistry in 4 culture dishes for each group. Most $(61.5 \% \pm 5 \%)$ of the SKMCs stained positively for MyoD1, and $93 \% \pm 4 \%$ of the VSMCs stained positively for $\alpha$-smooth muscle actin (Figure 3). Multinuclear myotubes were seen in all SKMC plates after differentiation in serum-free media.

\section{Function}

LV function was assessed 5 weeks after cell transplantation by means of isolated heart perfusion with a Langendorff preparation. Heart rate and coronary flow were not significantly different between normal media-injected control subjects and cell-transplantation groups at different balloon volumes (data not shown). Figure 4 demonstrates that LV systolic function was preserved by muscle cell transplantation from cardiomyopathic donors. Both peak systolic pressure and developed pressure were significantly greater after transplantation of VSMCs and SKMCs than that seen in media-injected control hearts. Diastolic function did not differ significantly among the 4 groups. Transplantation of cryopreserved SKMCs that appeared healthy in vitro resulted in a marked preservation of heart function similar to that seen in normal hamster hearts. In contrast, cryopreserved VSMCs that were abnormal in vitro had only a moderate beneficial effect on cardiac function after transplantation.

\section{Geometric Maps of the Left Ventricle}

LV geometry was assessed on the basis of computerized planimetry of heart sections. The SKMC- and VSMC-trans- 


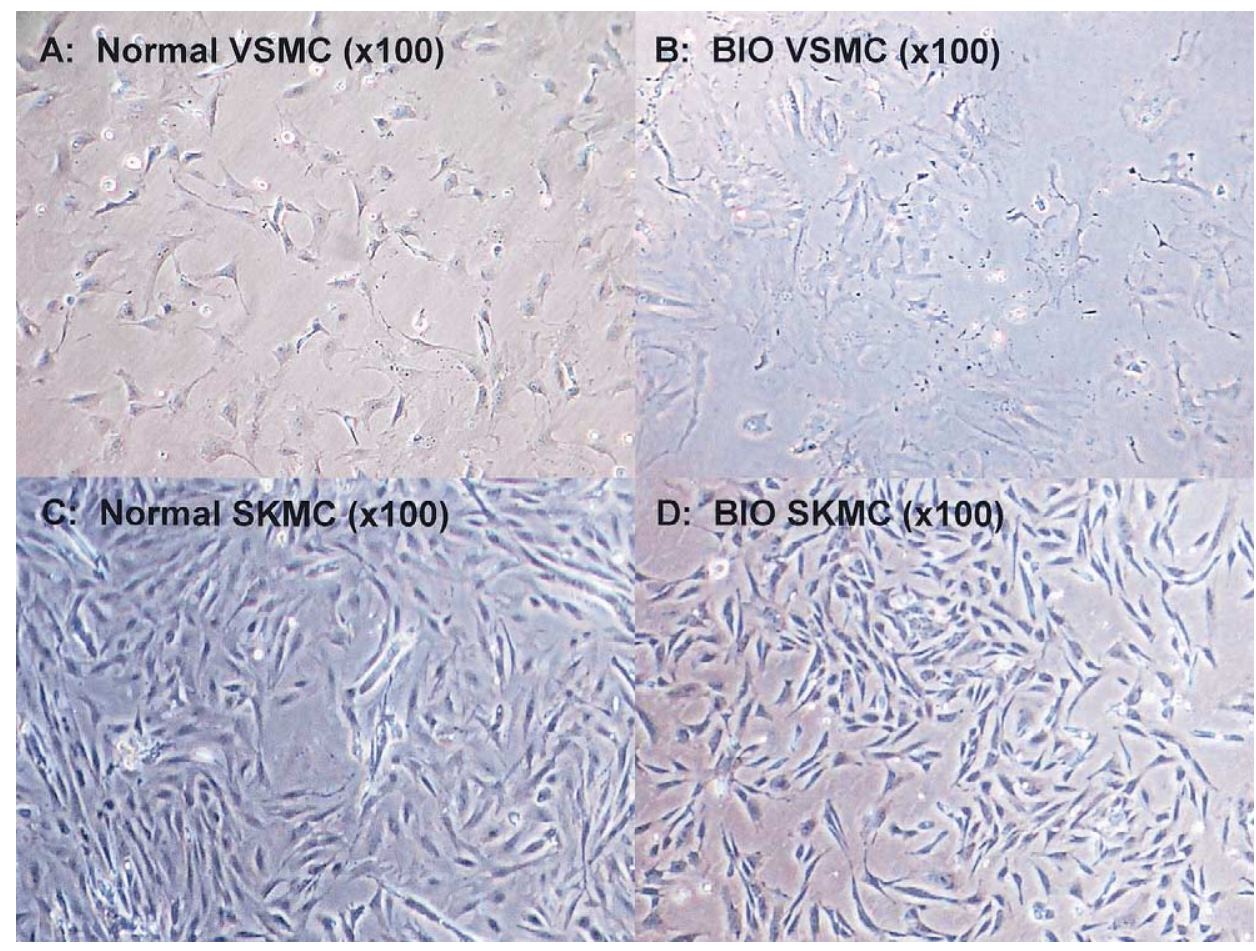

Figure 2. Photomicrographs of cultured VSMCs (A and B) and SKMCs (C and D) obtained from cryopreserved normal and cardiomyopathic hamsters. The normal spindle-shaped VSMCs contrasted in appearance with the cardiomyopathic VSMCS that were larger and not spindle shaped. The normal and cardiomyopathic SKMCs were similar in appearance.

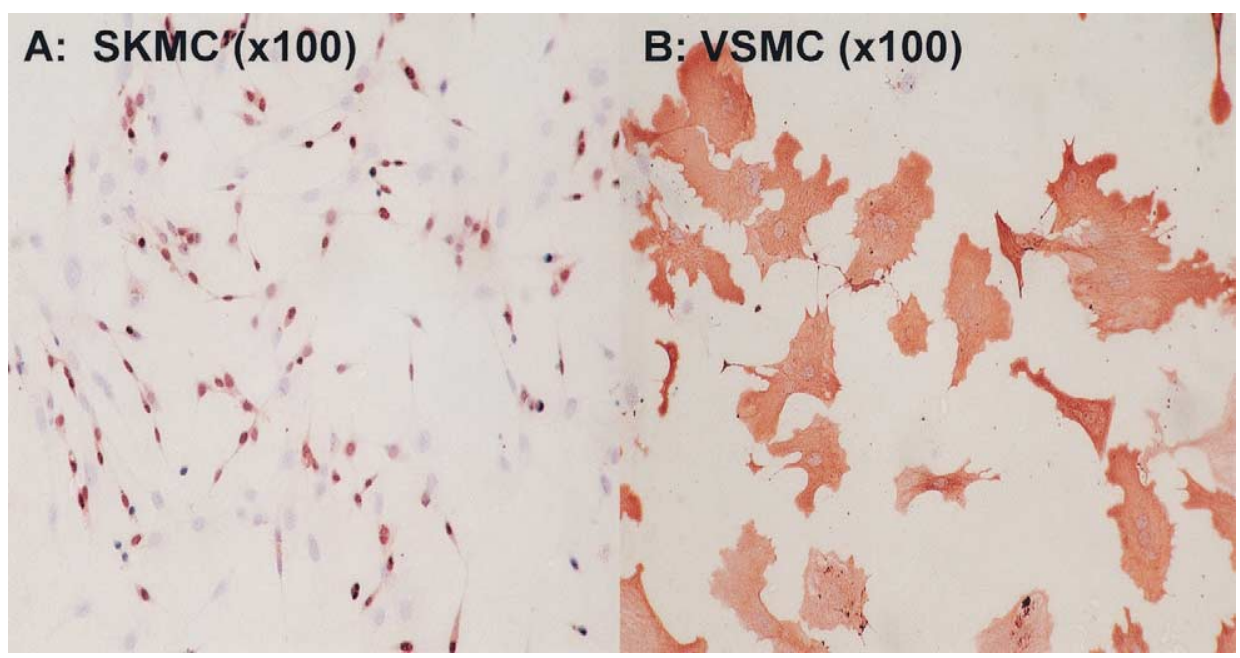

Figure 3. Photomicrographs of cultured cyropreserved BIO 53.58 SKMCs (A; original magnification 100X) and VSMCs (B; original magnification $100 \times$ ). The SKMCs stained positively for myoD, and the VSMCs stained positively for $\alpha$-smooth muscle actin.

planted chamber volumes $(65.9 \pm 5$ and $76.3 \pm 14 \mu \mathrm{L}$, respectively) were smaller (SKMCs, $P=.001$; VSMCs, $P$ $=.02)$ than those of media-injected control hamsters $(96.7$ $\pm 16 \mu \mathrm{L}$ ), indicating that cell transplantation limited ven- tricular dilatation and remodeling. Adverse chamber remodeling was attenuated most profoundly after transplantation of cryopreserved SKMCs, and chamber volumes approached those of age-matched normal hearts $(59.8 \pm 6$ 

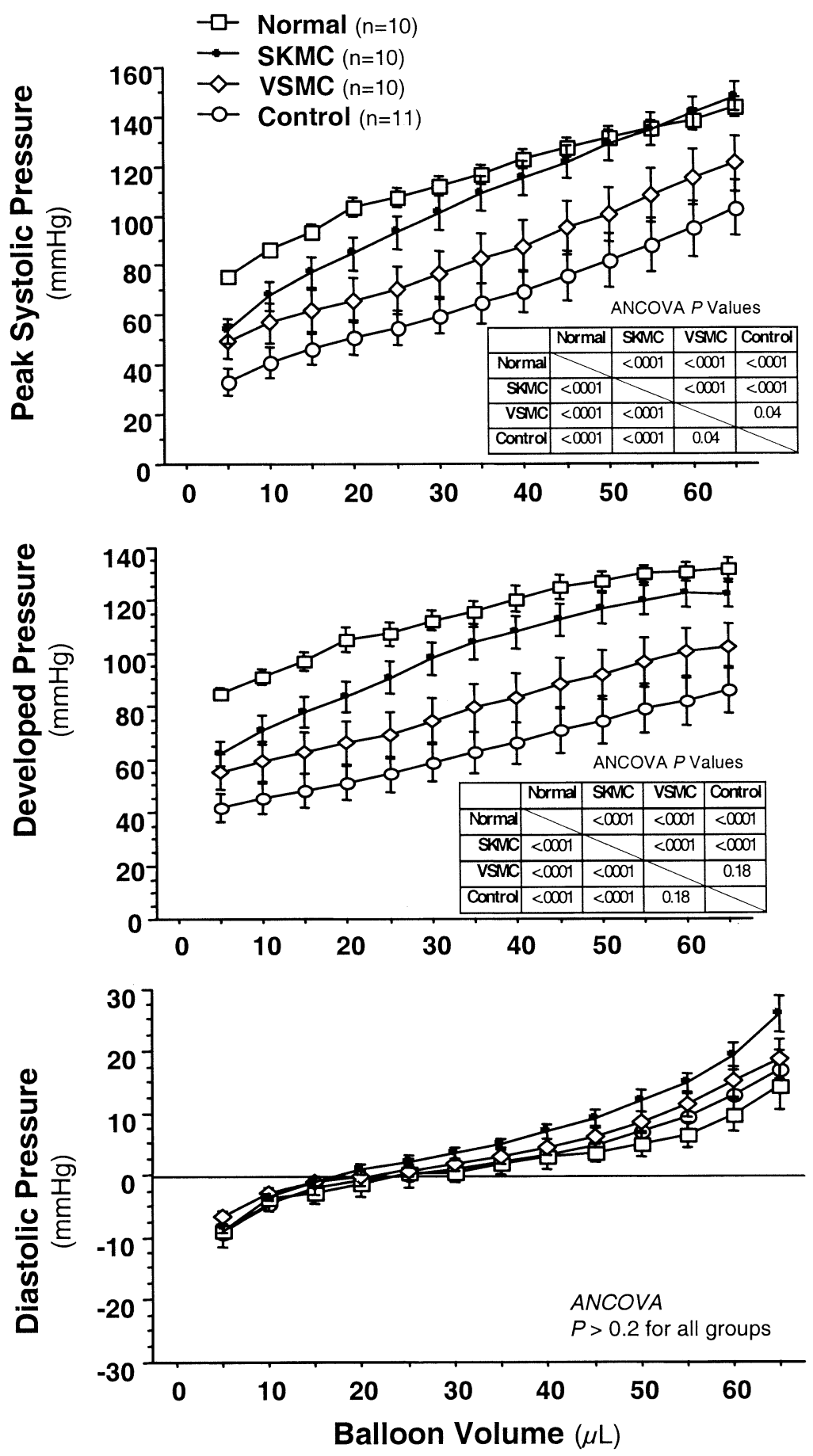

Figure 4. Peak systolic, developed, and end-diastolic pressures of normal, SKMC, VSMC, and control hearts with increasing balloon volumes. The peak systolic and developed pressures of the SKMC and normal hearts were significantly higher than the pressures of the VSMC hearts, which were greater than those of the control hearts. Diastolic function was not significantly different.

$\mu \mathrm{L})$. In addition, measurement of LV wall thickness at the level of the papillary muscles indicated that wall thinning was prevented by cell transplantation (Table 2).

\section{Histology}

As expected, all hearts from BIO 53.58 hamsters showed extensive myolysis, with dense replacement fibrosis char- 
TABLE 2. LV wall thickness (mm)

\begin{tabular}{lcccc}
\hline & $\begin{array}{c}\text { Normal } \\
(\mathbf{n}=\mathbf{7})\end{array}$ & $\begin{array}{c}\text { Control } \\
(\mathbf{n}=\mathbf{1 1 )}\end{array}$ & $\begin{array}{c}\text { VSMC } \\
(\mathbf{n}=\mathbf{1 0})\end{array}$ & $\begin{array}{c}\text { SKMC } \\
(\mathbf{n}=\mathbf{1 0})\end{array}$ \\
\hline Anterior wall & $2.2 \pm 0.2$ & $1.6 \pm 0.2^{*}$ & $2.1 \pm 0.4$ & $2.3 \pm 0.3$ \\
Posterior wall & $1.7 \pm 0.3$ & $1.4 \pm 0.1 \dagger$ & $1.8 \pm 0.4$ & $1.7 \pm 0.2$ \\
Septum & $1.4 \pm 0.2$ & $1.3 \pm 0.2$ & $1.5 \pm 0.3$ & $1.6 \pm 0.2 \ddagger$ \\
Lateral wall & $1.9 \pm 0.2$ & $2.0 \pm 0.3$ & $2.3 \pm 0.4 \S$ & $2.3 \pm 0.3 \|$ \\
Mean thickness & $1.8 \pm 0.2$ & $1.6 \pm 0.1 \uparrow$ & $1.9 \pm 0.2$ & $2.0 \pm 0.2$ \\
\hline
\end{tabular}

Wall thickness (mean \pm SD) was measured at the level the papillary muscles for the left ventricle.

The anterior, posterior, and lateral walls of the left ventricle in SKMC- and VSMC-transplanted hearts were thicker than the control walls.

${ }^{*} P<.001$ versus normal, $P<.001$ versus VSMC, $P<.001$ versus SKMC.

$\dagger P=.005$ versus normal, $P<.001$ versus VSMC, $P=.002$ versus SKMC.

$\ddagger P=.002$ versus control.

$\S P=.04$ versus normal.

$\| P=.03$ versus normal, $P=.045$ versus control.

$\Uparrow P<.001$ versus VSMC, $P<.001$ versus SKMC.

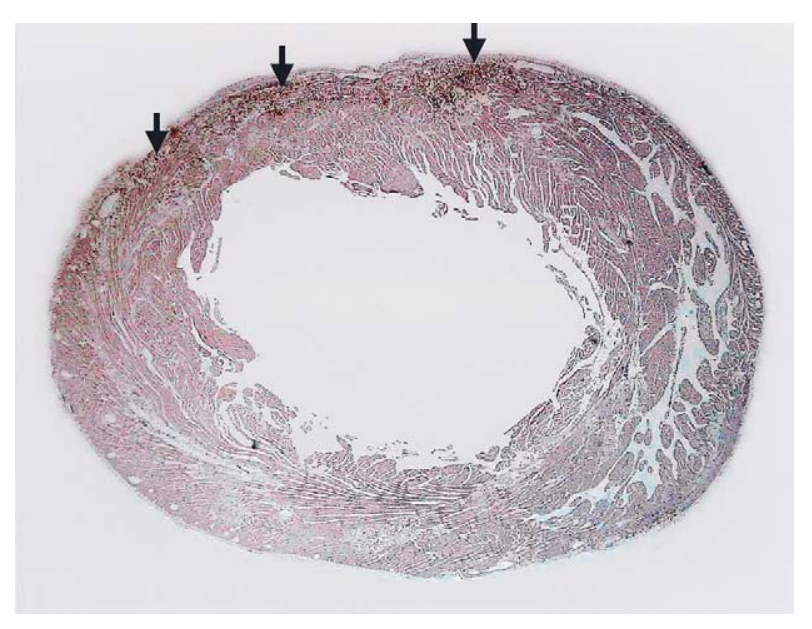

Figure 5. Location of bromodeoxyuridine-labeled SKMCs transplanted in the anterior left ventricle of the cardiomyopathic BIO 53.58 hamster. The labeled cells (arrows) were identified only in the LV anterior wall.

acteristic of the underlying cardiomyopathic process. These changes were absent in normal hearts. Transplantation of both SKMCs and VSMCs resulted in the formation of muscle tissue in the anterior aspect of the left ventricle (Figure 5). The engrafted cells were evident by means of hematoxylin and eosin staining (Figure 6, $A$ and $B$ ) and confirmed as the transplanted cells by means of BrdU staining (Figure 6,C and $D$ ). The SKMCs were found in both the subepicardial and midmyocardial regions, whereas VSMCs localized to the subepicardial layer. Although transplanted cells were observed in the heart after a period of 5 weeks, the VSMC transplantation formed a thin and sparsely populated tissue, as assessed by means of BrdU staining. This suggests limited engraftment with VSMCs compared with SKMCs. Elastin Masson trichrome staining (Figure 6, $E$ and $F$ ) demonstrated an extensive extracellular elastin matrix surrounding the SKMCs compared with the VSMCs. In contrast, dense fibrillar collagen rather than an organized extracellular matrix surrounded the VSMCs. In addition, the engraftment of these allogeneic cells was associated with a mild lymphocytic infiltration (Figure 6, $A$ and $B$ ), suggesting that a chronic process of mild rejection occurred despite daily cyclosporine treatment. The engrafted SKMCs stained positively for muscle-specific actin (Figure 7, $A$ ) and skeletal muscle fast myosin heavy chain (Figure $7, B$ ) but did not stain for cardiac $\beta$-myosin heavy chain (Figure 7, $C$ ). The engrafted VSMCs stained positively for $\alpha$-smooth muscle actin (Figure 7, D).

\section{Discussion}

This study offers the following observations: (1) extracardiac muscle cells can be isolated, cultured, cryopreserved, and stored for at least 3 weeks and injected into a cardiomyopathic heart, and the cells will engraft, limit cardiac thinning and dilatation, and preserve global systolic function for at least 5 weeks; (2) donor cells that exhibit morphologic abnormalities and decreased growth in vitro result in diminished engraftment and less successful preservation of ventricular size and function; and (3) with inherited cardiomyopathies, some peripheral muscle cells might also be affected by the myopathic process limiting their beneficial effects after cell transplantation. These insights might be helpful in designing clinical trials intended to limit cardiac thinning and dilatation in patients with an inherited dilated cardiomyopathy.

\section{Cell Types}

We previously demonstrated that the transplantation of allogeneic heart cells and autologous smooth muscle cells prevented cardiac thinning and dilatation and preserved systolic ventricular function similar to that seen in other cell types. ${ }^{7,8}$ We used heart cells from young (4-week-old) BIO 53.58 hamsters before cardiomyopathy developed. Our pre- 


\section{SKMC}

\section{VSMC}

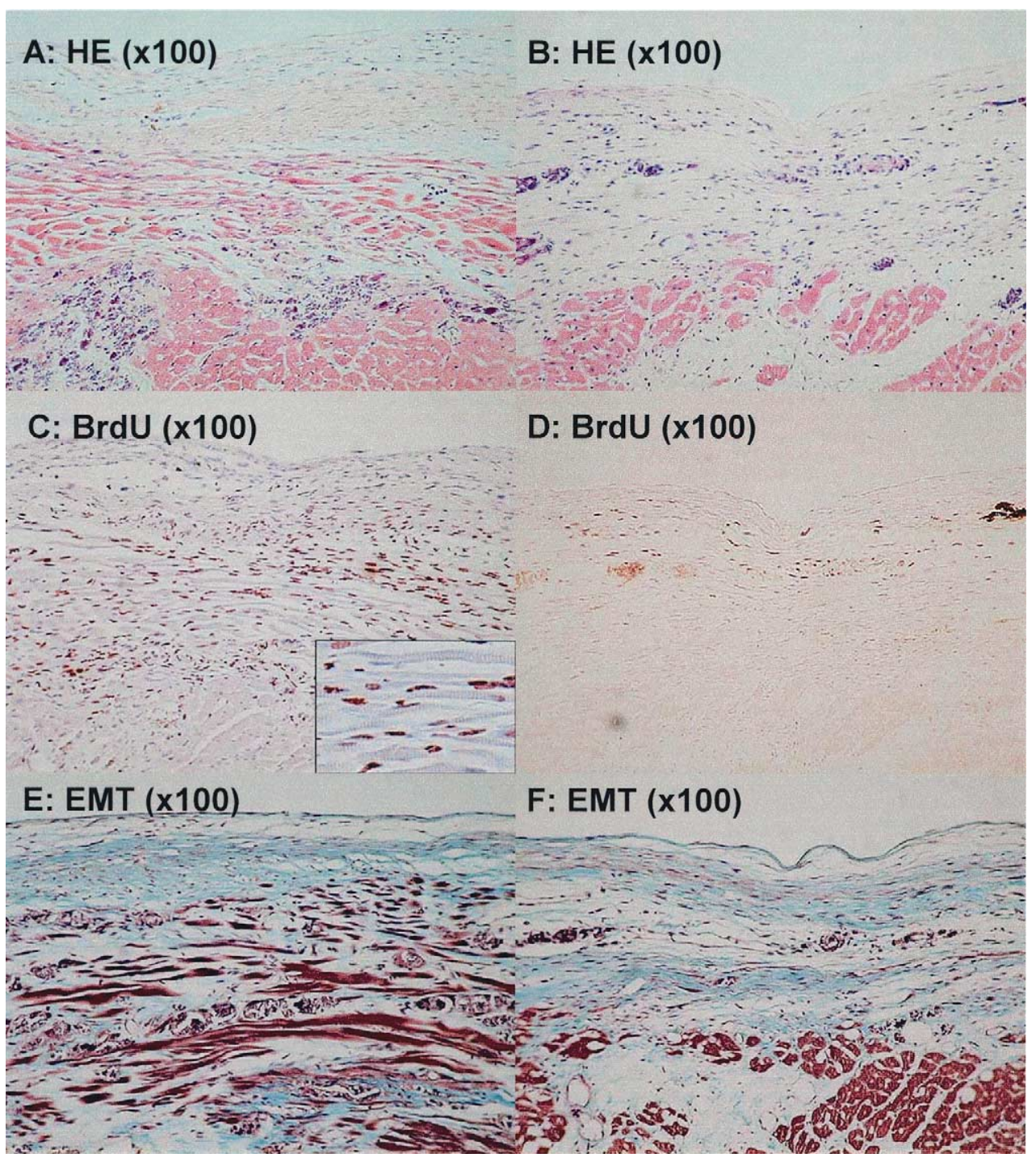

Figure 6. Hematoxylin and eosin-stained (HE), anti-bromodeoxyuridine-stained (BrdU), and elastica Masson trichrome-stained (EMT) SKMC and VSMC transplants in the host BIO 53.58 hamster LV anterior wall. The SKMC transplant contained many BrdU-labeled cells (C), was substantial, and had less fibrosis and more elastin (A and E) than the VSMC transplant (B, D, and F). Some of the SKMCs had mature sarcomeres (inset, C). The VSMC transplant tended to be localized to the subepicardium, whereas the SKMC transplant could be found in the subepimyocardium and midmyocardium.

vious study established a proof of concept, but heart cell transplantation might not be clinically relevant if the heart cells are abnormal and grow slowly ex vivo. Patients with a progressive dilated cardiomyopathy could have abnormal heart cells, which might not be suitable for transplantation. Autologous smooth muscle cells might be ideal donor cells to prevent congestive heart failure. Smooth muscle cells respond to hemodynamic stresses by means of hyperplasia and hypertrophy, and they rapidly establish an extracellular matrix that communicates with the surrounding viable cardiomyocytes. Therefore smooth muscle cells might be ideal donor cells for cardiac regeneration. We previously demonstrated that stomach smooth muscle cells engrafted in a cryoinjured cardiac scar and preserved ventricular function. ${ }^{3}$ These cells responded to the hemodynamic stresses 4 weeks after transplantation by means of hyperplasia, but not hypertrophy, to maintain wall thickness. ${ }^{3}$ Autologous smooth muscle cells from the ductus deferens of adult 




Figure 7. Immunohistochemical staining of the SKMC and VSMC transplants. The SKMC transplant stained positively for muscle-specific actin (MSA; $A)$ and skeletal muscle fast myosin heavy chain $(S M H C ; B)$ and negatively for $\beta$-myosin heavy chain $(\beta M H C ; C)$. The VSMC transplant stained positively for $\alpha$-smooth muscle actin $(\alpha S M A ; D)$. Arrows indicate positive staining.

(13-week-old) BIO 53.58 hamsters were expanded rapidly ex vivo and engrafted in the anterior wall of cardiomyopathic hamsters after transplantation. ${ }^{8}$ Cell engraftment prevented cardiac dilatation and preserved ventricular function. However, harvesting cells from the vas deferens might be inconvenient in male patients and not possible in female patients. Therefore in the present study we evaluated VSMCs, which could be harvested from a peripheral vein in patients at an early stage of cardiomyopathy. We used allogeneic aortic smooth muscle cells from the cardiomyopathic hamsters to mimic an autologous transplant. The small size of the animals precluded an actual autologous transplantation of VSMCs. Interestingly, we found that the VSMCs from cardiomyopathic donors had an abnormal morphology and a restricted growth pattern. This was not the case for SKMCs or VSMCs from normal donors.

The BIO 53.58 hamster has a muscular dystrophy from an inherited $\delta$-sarcoglycan gene deficiency, ${ }^{13,14}$ which involves cardiac, skeletal, and smooth muscle to varying degrees. The phenotype is most pronounced in mature VSMCs as a result of disruption of the sarcoglycan-sarcospan complex found only in vascular smooth muscle. ${ }^{10}$ In fact, microvascular spasm resulting in diffuse microinfarcts is believed to mediate the cardiomyopathy associated with this genetic defect. ${ }^{15,16}$ In our study the abnormal VSMCs grew slowly ex vivo, and this characteristic predicted the limited engraftment of these cells in the anterior wall of the cardiomyopathic hamsters. These slow-growing cells produced fewer engrafted cells and induced only a modest improvement in systolic function. These results suggest that ex vivo morphology and growth rates can be used to predict the success of subsequent cell transplantation. In some patients with a cardiomyopathy, VSMCs might be normal, and results comparable with those of the SKMC are anticipated. In support, normal VSMCs have been successfully used for the creation of autologous vascular grafts. ${ }^{17}$

\section{Cryopreservation}

Both SKMCs and VSMCs successfully limited cardiac dilatation and preserved ventricular function after cryopreservation for 3 weeks. However, the tolerance of the cells to cryopreservation was different. Donor cells showing abnormalities in vitro were further compromised after a period of cryostorage. These results suggest that the clinical application of cryopreservation for storage of donor cells should be used with caution if the cells show abnormal morphology or poor growth in the initial culture period. In a previous study fetal cardiomyocytes proliferated and beat in vitro and en- 
grafted into the cryoinjured heart after 4 weeks of cryopreservation. ${ }^{18}$ However, in both studies cryopreservation decreased the rate of proliferation and probably reduced cell survival after cardiac implantation. Cryopreservation might permit elective cell transplantation early in the course of cardiac decompensation in patients with a cardiomyopathy. However, more cells might need to be implanted after cryopreservation to obtain the same degree of enhancement of ventricular function.

\section{Improvement in Ventricular Function}

The mechanism by which SKMCs and VSMCs preserved ventricular function remains obscure. Previous studies have not conclusively identified synchronous contraction of the SKMCs implanted in injured myocardial regions. Therefore active cellular contraction is unlikely to explain the preserved systolic function seen after cell engraftment. In our study the implanted cells established a rich extracellular matrix, with connections to the remaining viable cardiomyocytes. Improvements in function could have resulted from the cells actively establishing an organized interstitial matrix, which limited dilatation and thinning. Importantly, the apparent health of the cells in vitro predicted the degree of engraftment and the subsequent benefits on cardiac structure and function after transplantation. These data indicate that the health of the donor cells influences the process of cellular engraftment and suggest that the benefits of cell transplantation are dependent on the number of cells that successfully engraft in the host myocardium. Although wall thickness increased equally by using the different cell types transplanted, the SKMCs were healthy in culture and afforded greater benefits on limiting dilatation and preserving cardiac function than the abnormal VSMCs. These data might imply that transplanted cells limit remodeling in the failing heart as a result of active cellular events after engraftment and not as a result of passive ventricular constraint from increased wall thickness.

\section{Clinical Implications}

Cryopreserving donor cells might allow for targeted cell therapy at predetermined intervals in the progression of global cardiac failure. For example, for patients with compensated dilated cardiomyopathy, targeted therapy at the onset of significant chamber dilatation and increased wall stress might prevent decompensation that would otherwise lead to whole organ transplantation, ventricular device assistance, or death. With the aid of cryopreservation, harvest of muscle cells when the patient is both physiologically and pharmacologically stable will be possible. This available pool of autologous donor cells will be available for a second transplantation if decompensation should occur. After a period of hemodynamic unloading while on mechanical assistance, cell transplantation might provide a stabilizing addition to the transient benefits offered by the process of reverse remodeling. Although the specific molecular effects of cryopreservation on donor cells were not examined, in this study the important clinical benefits on myocardial remodeling and cardiac function were not lost as a result of donor cell cryostorage. Our initial observations have now been extended in a more clinically useful framework that favors the future use of cryopreservation for targeted cell therapy.

\section{Limitations and Summary}

Some of our study's limitations deserve mention. We were forced to explore allotransplantation of noncardiac muscle cells because the small size of the hamsters made autotransplantation of vascular smooth muscle difficult. Despite the use of cyclosporine, rejection was not completely eliminated, and this might have influenced the assessment of engraftment. However, the ability of the allogeneic noncardiac muscle cells to engraft and remain viable in diseased myocardium under hemodynamic strain after prior cryostorage makes the prospect of similar autogenous cells even more promising. Assessment of LV function by the use of the Langendorff perfusion apparatus has been criticized in the past, but echocardiography and in vivo catheter assessment in such a small-animal model has not proved reliable in our hands. The lack of specific data on the differential effects of cryopreservation on the cell types used in the study make interpretation of the results more difficult, a problem that could be addressed in future studies. Additionally, given that the mechanism by which transplanted cells influence cardiac remodeling and function is unknown, interpretation of the data is limited. Future studies to uncover the specific molecular and cellular effects of cell transplantation in globally dysfunctional myocardium are necessary before this novel therapy can become a substantive clinical approach for heart failure.

In summary, noncardiac muscle cells from cardiomyopathic donors can be successfully expanded in culture, cryopreserved, and later transplanted with significant engraftment at 5 weeks, despite an ongoing cardiomyopathic process. Cryopreserved SKMCs and VSMCs maintained chamber geometry and cardiac function in failing hearts after transplantation. These data suggest a clinically applicable and effective approach for ventricular restoration with cell-transplantation therapy for patients with inherited dilated cardiomyopathy.

\section{References}

1. Jain M, DerSimonian H, Brenner DA, Ngoy S, Teller P, Edge AS, et al. Cell therapy attenuates deleterious ventricular remodeling and improves cardiac performance after myocardial infarction. Circulation. 2001;103(14):1920-7.

2. Li RK, Jia ZQ, Weisel RD, Mickle DA, Zhang J, Mohabeer MK, et al. Cardiomyocyte transplantation improves heart function. Ann Thorac Surg. 1996;62(3):654-60. 
3. Li RK, Jia ZQ, Weisel RD, Merante F, Mickle DA. Smooth muscle cell transplantation into myocardial scar tissue improves heart function. J Mol Cell Cardiol. 1999;31(3):513-22.

4. Li RK, Weisel RD, Mickle DA, Jia ZQ, Kim EJ, Sakai T, et al. Autologous porcine heart cell transplantation improved heart function after a myocardial infarction. J Thorac Cardiovasc Surg. 2000;119(1): 62-8.

5. Rajnoch C, Chachques JC, Berrebi A, Bruneval P, Benoit MO, Carpentier A. Cellular therapy reverses myocardial dysfunction. $J$ Thorac Cardiovasc Surg. 2001;121(5):871-8.

6. Watanabe E, Smith DM Jr, Delcarpio JB, Sun J, Smart FW, Van MC $\mathrm{Jr}$, et al. Cardiomyocyte transplantation in a porcine myocardial infarction model. Cell Transplant. 1998;7(3):239-46.

7. Yoo KJ, Li RK, Weisel RD, Mickle DA, Jia ZQ, Kim EJ, et al. Heart cell transplantation improves heart function in dilated cardiomyopathic hamsters. Circulation. 2000;102(suppl 3):III204-9.

8. Yoo KJ, Li RK, Weisel RD, Mickle DA, Li G, Yau TM. Autologous smooth muscle cell transplantation improved heart function in dilated cardiomyopathy. Ann Thorac Surg. 2000;70(3):859-65.

9. Scorsin M, Hagege AA, Dolizy I, Marotte F, Mirochnik N, Copin H, et al. Can cellular transplantation improve function in doxorubicininduced heart failure? Circulation. 1998;98(suppl):II151-5.

10. Cohn RD, Durbeej M, Moore SA, Coral-Vazquez R, Prouty S, Campbell KP. Prevention of cardiomyopathy in mouse models lacking the smooth muscle sarcoglycan-sarcospan complex. J Clin Invest. 2001; 107(2):R1-7.

11. Pfeffer JM, Pfeffer MA, Fletcher PJ, Braunwald E. Progressive ven- tricular remodeling in rat with myocardial infarction. Am J Physiol. 1991;260(suppl):H1406-14.

12. Jugdutt BI, Joljart MJ, Khan MI. Rate of collagen deposition during healing and ventricular remodeling after myocardial infarction in rat and dog models. Circulation. 1996;94(1):94-101.

13. Nigro V, Okazaki Y, Belsito A, Piluso G, Matsuda Y, Politano L, et al. Identification of the Syrian hamster cardiomyopathy gene. Hum Mol Genet. 1997;6(4):601-7.

14. Sakamoto A, Ono K, Abe M, Jasmin G, Eki T, Murakami Y, et al. Both hypertrophic and dilated cardiomyopathies are caused by mutation of the same gene, delta-sarcoglycan, in hamster: an animal model of disrupted dystrophin-associated glycoprotein complex. Proc Natl Acad Sci U S A. 1997;94(25):13873-8.

15. Coral-Vazquez R, Cohn RD, Moore SA, Hill JA, Weiss RM, Davisson RL, et al. Disruption of the sarcoglycan-sarcospan complex in vascular smooth muscle: a novel mechanism for cardiomyopathy and muscular dystrophy. Cell. 1999;98(4):465-74.

16. Straub V, Ettinger AJ, Durbeej M, Venzke DP, Cutshall S, Sanes JR, et al. epsilon-sarcoglycan replaces alpha-sarcoglycan in smooth muscle to form a unique dystrophin-glycoprotein complex. J Biol Chem. 1999;274(39):27989-96.

17. Shin'oka T, Imai Y, Ikada Y. Transplantation of a tissue-engineered pulmonary artery. $N$ Engl J Med. 2001;344(7):532-3.

18. Yokomuro H, Li RK, Mickle DA, Weisel RD, Verma S, Yau TM. Transplantation of cryopreserved cardiomyocytes. J Thorac Cardiovasc Surg. 2001;121(1):98-107. 